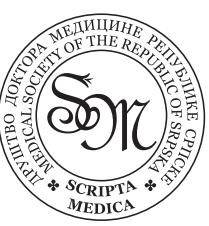

CASE REPORT

\title{
A Young Woman with a Swollen and Tender Umbilical Nodule
}

August A. Natalie, Anjeli K. Isaac

Department of Medicine, Division of Dermatology, John H. Stroger, Jr. Hospital of Cook County, Chicago, Illinois 60612, USA

\section{Correspondence}

August A. Natalie, MD

John H. Stroger Jr. Hospital of Cook County

Department of Internal Medicine

Division of Dermatology

Chicago, Illinois 60612

USA

Email: anatalie@gmail.com

Submitted: April 2, 2011

Accepted: May 5, 2011
A 20-year-old woman presented with a six-month history of a swollen and tender umbilical nodule (Figure 1A). The patient reported intermittent pain in that area since age nine. She recalled monthly episodes of umbilical swelling and bleeding, followed by regression. The pain was worse with sitting and at times persisted throughout the day. She also noted recurrent tender subcutaneous nodules in the periumbilical area and within abdominal striae. Menarche began at age ten. She had regular menstrual periods every 23 days, but they were complicated by significant menorrhagia. Review of systems was notable for dyspnea, a sensation of something pushing up on the diaphragm, chronic burning in the lower abdomen, alternating diarrhea and constipation, infertility for the past18 months, and intermittent but severe back pain. An abdominal ultrasound performed by the primary care physician was negative for umbilical hernia. A punch biopsy of the umbilical nodule was obtained (Figure 1B and $\mathbf{1 C}$ ).

The skin biopsy specimen showed two small isolated atypical glands with ciliated columnar epithelium surrounded by scant stroma. There was no evidence of granulomas, hemorrhage, hemosiderin or fecal material. A diagnosis of cutaneous endosalpingiosis was made. The patient was offered excision of the umbilical nodule, but she opted to try medical management first. She was referred to the gynecology-endocrinology department for evaluation and was started on leuprolide, a gonadotropin-releasing hormone (GnRH) agonist.

Endosalpingiosis is the aberrant growth of Fallopian tube epithelium outside of its usual location. ${ }^{1}$ Endometriosis and endosalpingiosis are similar disease entities that share common clinical features. There is a paucity of information regarding endosalpingiosis in the literature; thus, the management of endosalpingiosis derives from the literature on endometriosis. Endometriosis (ectopic endometrial tissue) is an estrogen-dependent inflammatory disease that affects $5 \%$ to $10 \%$ of women of reproductive age in the United States. ${ }^{2}$ The main clinical features of endometriosis are pelvic pain, dysmenorrhea, dyspareunia, cyclical bowel or bladder symptoms and infertility. ${ }^{3}$ It is well known that endometriosis can produce skin lesions, with a predilection for abdominal scars ${ }^{3}$ and the umbilicus. ${ }^{4}$ The incidence of cutaneous endometriosis is between $1.1 \%^{3}$ and $5.2 \% .^{5} \mathrm{En}$ dometriosis of the umbilicus (also known as a Villar's nodule) is a rare clinical finding with an estimated incidence of $0.5 \%$ to $1 \%$ of all patients with endometrial ectopia. ${ }^{6}$

Umbilical endometriosis usually follows laparoscopic surgery; however, spontaneous lesions have been reported as well. ${ }^{4}$ Umbilical endosalpingiosis appears to be an even rarer disease. To our knowledge, only four cases of cutaneous endosalpingiosis of the umbilicus have been reported. ${ }^{1}$ ${ }^{7,8} \mathrm{An}$ umbilical nodule requires biopsy in order to rule out the possibility of malignant melanoma or a Sister Mary Joseph nodule (metastasis usually from an intra-abdominal tumor).

There are three different theories concerning the pathogenesis of endosalpingiosis. These are similar to the pathogenesis of endometriosis: metaplasia, implantation and metastasis. ${ }^{1}$ According to the metaplasia theory, the peritoneum retains the potential for differentiation into various types of epithelium, including endometrial tissue. The implantation theory suggests that retrograde passage 

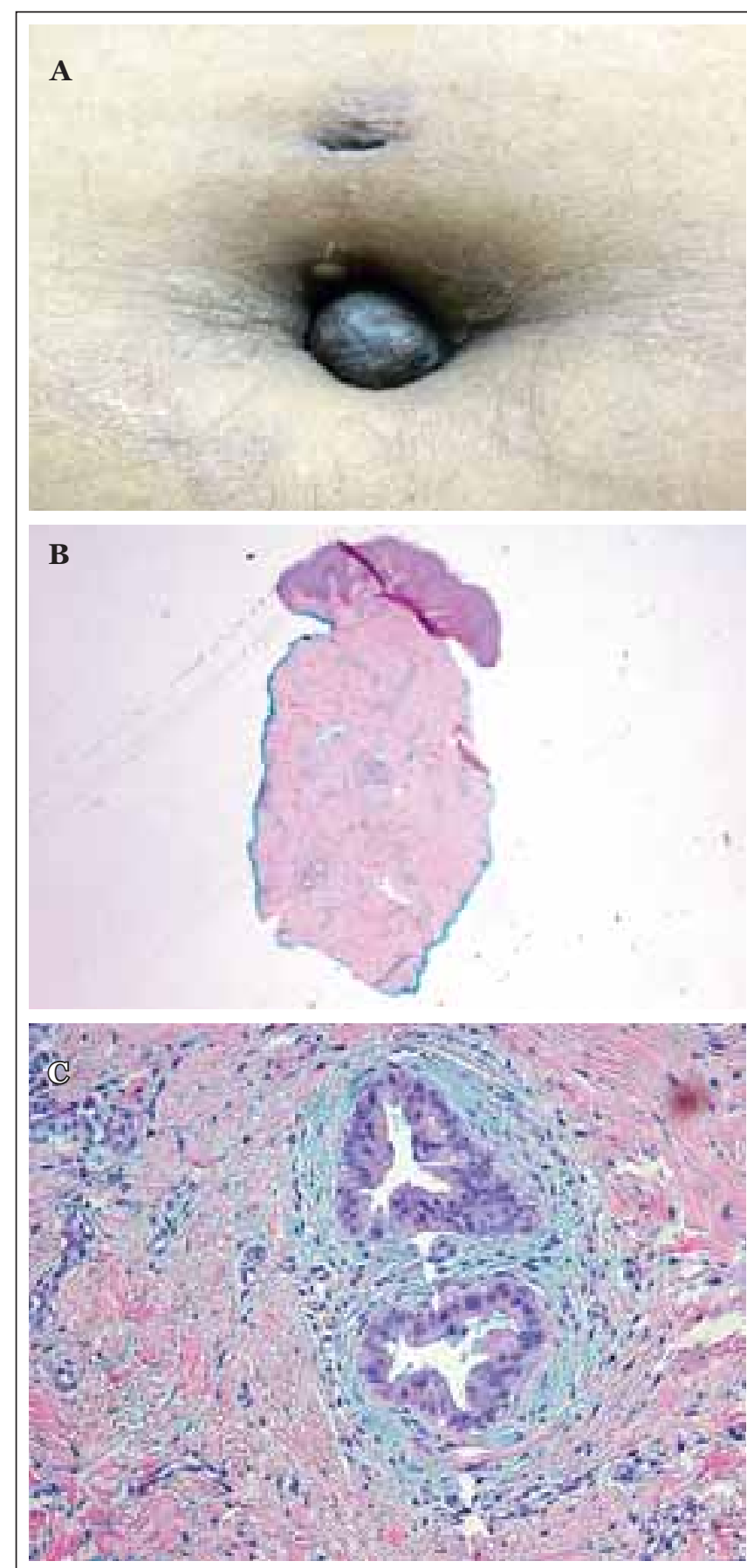

Figure 1. Umbilical nodule. A. Umbilical swelling, B. A punch biopsy of the umlical nodule, C. Skin biopsy-tvo small isolated atypical glands. of tubal tissue during menstruation can implant on pelvic structures. Finally, the metastatic theory suggests that salpingeal tissue spreads via vascular or lymphatic vessels.

The mainstays of treatment for endometriosis are medication and/or surgery, depending on whether therapy is being directed toward pelvic pain or infertility. ${ }^{9}$ Both medical and surgical treatments are effective for treatment of pelvic pain. Therapeutic options include NSAIDs, oral contraceptives, or GnRH-agonist therapy, and if these prove unsuccessful, laparoscopy. For infertility associated with endometriosis, medical treatment is ineffective, but surgical removal of endometrial tissue is beneficial for most women.

Our patient received seven injections of leuprolide $3.75 \mathrm{mg}$ IM monthly. After two injections, she noted cessation of the umbilical bleeding. Eight months after beginning leuprolide, she underwent a diagnostic laparoscopy with chromopertubation that revealed a normal uterus and ovaries; the fallopian tubes were open with normal flow of methylene blue from the fimbriae. Four months later, umbilical bleeding and painful menstrual periods resumed. She has been since referred to general surgery for umbilical tissue removal.

\section{REFERENCES}

1. Dore N, Landry M, Cadotte M, et al. Cutaneous Endosalpingiosis. Arch Dermatol 1980;116:909-12.

2. Bulun SE. Endometriosis. N Engl J Med 2009;360:268-79.

3. Agarwal A, Fong YF. Cutaneous endometriosis. Singapore Med $J$ 2008;49:704-9.

4. Chatzikokkinou P, Thorfinn J, Angelidis IK, et al. Spontaneous endometriosis in an umbilical skin lesion. Acta Dermatovenerol Alp Panonica Adriat 2009;18:126-30.

5. Douglas C, Rotimi O. Extragenital endometriosis--a clinicopathological review of a Glasgow hospital experience with case illustrations. J Obstet Gynaecol 2004;24:804-8.

6. Dessy LA, Buccheri EM, Chiummariello S, et al. Umbilical endometriosis, our experience. In Vivo 2008;22:811-5.

7. Perera GK, Watson KM, Salisbury J, et al. Two cases of cutaneous umbilical endosalpingiosis. Br J Dermatol 2004;151:924-5.

8. Redondo P, Idoate M, Corella C. Cutaneous umbilical endosalpingiosis with severe abdominal pain. $J$ Eur Acad Dermatol Venereol 2001;15:179-80.

9. Olive DL, Pritts EA. Treatment of endometriosis. N Engl J Med 2001;345:266-75. 